\title{
Dark Matter Freeze-Out via Catalyzed Annihilation
}

\author{
Chuan-Yang Xing $\circledast^{1, *}$ and Shou-hua Zhu ${ }^{1,2,3, \dagger}$ \\ ${ }^{1}$ Department of Physics and State Key Laboratory of Nuclear Physics and Technology, Peking University, \\ Beijing 100871, China \\ ${ }^{2}$ Collaborative Innovation Center of Quantum Matter, Beijing, 100871, China \\ ${ }^{3}$ Center for High Energy Physics, Peking University, Beijing 100871, China
}

(Received 20 February 2021; revised 31 May 2021; accepted 12 July 2021; published 6 August 2021)

\begin{abstract}
We present a new paradigm of dark matter freeze-out, where the annihilation of dark matter particles is catalyzed. We discuss in detail the regime in which the depletion of dark matter proceeds via $2 \chi \rightarrow 2 A^{\prime}$ and $3 A^{\prime} \rightarrow 2 \chi$ processes, where $\chi$ and $A^{\prime}$ denote dark matter and the catalyst, respectively. In this regime, the dark matter number density is depleted polynomially rather than exponentially (Boltzmann suppression) as in classical weakly interacting massive particles and strongly interacting massive particles. The paradigm applies for a secluded weakly interacting dark sector with dark matter in the MeV-TeV mass range. The catalyzed annihilation paradigm is compatible with cosmic microwave background and big bang nucleosynthesis constraints, with enhanced indirect detection signals.
\end{abstract}

DOI: 10.1103/PhysRevLett.127.061101

Introduction.-The existence of dark matter (DM) is well established via ample evidence from cosmological and astrophysical observations [1]. However, the nature of dark matter is still unknown. To solve this puzzle, tremendous efforts have been devoted to searching for dark matter candidates and studying production mechanisms of dark matter in the early Universe. Among all the mechanisms that reproduce the observed abundance of dark matter, the possibility of thermal dark matter, where dark matter keeps in thermal equilibrium with standard model (SM) particles in the early Universe, is especially popular and compelling.

For massive thermal dark matter, DM particles remain in thermal and chemical equilibrium while dark matter particles are relativistic. As the universe cools down, DM particles are depleted via certain processes, and the abundance of dark matter goes down. These processes "freeze out" when their interaction rate falls below the expansion rate of the Universe, and consequently the DM abundance stops decreasing and is a constant afterwards. There are essentially two kinds of processes leading to depletion of DM particles described in the literature. The first type is the process in which DM particles annihilate into other particles, mostly SM particles. The other type is the number-changing process of dark matter. For the first case, the most studied scenario is the self-annihilation process [2], e.g., $2 \mathrm{DM} \rightarrow 2 \mathrm{SM}$. In particular, weakly interacting

Published by the American Physical Society under the terms of the Creative Commons Attribution 4.0 International license. Further distribution of this work must maintain attribution to the author(s) and the published article's title, journal citation, and DOI. Funded by SCOAP. massive particles (WIMPs) that naturally reproduce the correct relic abundance have attracted extensive attention [3-5]. Other variations of the self-annihilation case include coannihilation [6-8], semiannihilation [9], and the like [6,10-21]. For the number-changing process, the most studied process is $3 \mathrm{DM} \rightarrow 2 \mathrm{DM}$ annihilation, dubbed as strongly interaction massive particle (SIMP) annihilation $[22,23]$. Subsequently, other number-changing processes have been proposed and discussed, including the $Z_{2}$-symmetric SIMP [24,25] and co-SIMP processes [26].

In this Letter, we propose a new pattern of dark matter burning in the early Universe beside the two aforementioned kinds of processes, where the abundance of dark matter is determined by "catalyzed" processes. In catalyzed processes, there are some other particles beside DM that act as the catalyst. The catalyst can enhance the rate of dark matter burning, yet the catalyst itself is not consumed in the reaction [27-29]. This process provides us with an alternative reaction pathway to make the reaction happen without changing the reactants and products. Specifically, in this work we study a simple regime of catalyzed annihilation with two processes leading to depletion of DM particles: $2 \chi \rightarrow 2 A^{\prime}$ and $3 A^{\prime} \rightarrow 2 \chi$, where $\chi$ and $A^{\prime}$ denote dark matter and the catalyst, respectively. We show in Fig. 1 a depiction of how these annihilation channels result in the depopulation of DM particles, that is, three $2 \chi \rightarrow 2 A^{\prime}$ processes together with two $3 A^{\prime} \rightarrow 2 \chi$ processes effectively deplete two DM particles. Note that the assisted annihilations $[20,21,30]$ are not catalyzed reactions since the assisters are consumed in the reaction. The co-SIMP process $\mathrm{SM}+\chi+\chi \rightarrow \mathrm{SM}+\chi$ [26] is not a catalyzed reaction either, since $\chi+\chi \rightarrow \chi$ is kinetically forbidden and it is groundless to discuss 


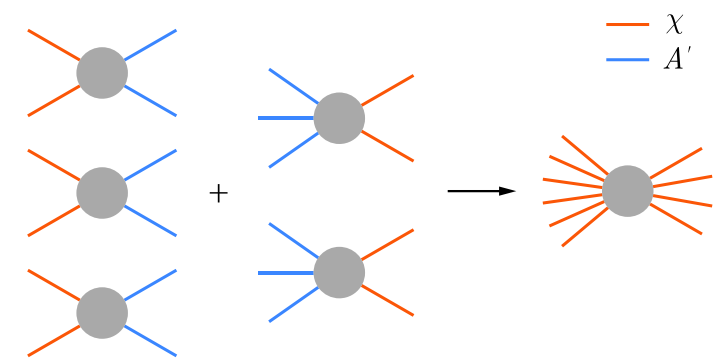

FIG. 1. Schematic illustration of the catalyzed annihilation of DM $\chi$ (red line) with a catalyst $A^{\prime}$ (blue line). Three $2 \chi \rightarrow 2 A^{\prime}$ processes plus two $3 A^{\prime} \rightarrow 2 \chi$ processes effectively deplete the number of DM particles by two.

enhancement of this unphysical process. The same thing happens to Ref. [31]. We note also that catalyzed processes are being considered in big bang nucleosynthesis [32].

The observed DM abundance can be reproduced in the catalyzed annihilation paradigm for a wide mass range of dark matter. We emphasis that the thermal evolution in the catalyzed annihilation paradigm is unique. Unlike WIMPs and SIMPs, where the DM number density $n_{\gamma}$ tracks the Boltzmann distribution and shrinks exponentially before freeze-out, the catalyzed annihilation could lead to this polynomial suppression of $n_{\chi}$ as the Universe cools down:

$$
n_{\chi} \propto s^{3 / 2} \propto T^{9 / 2}
$$

where $s$ and $T$ denote the entropy density and temperature of the Universe. Thus, the catalyzed annihilation lasts longer and freezes out at later times. To reproduce a correct relic abundance, the cross section of DM annihilation $2 \chi \rightarrow 2 A^{\prime}$ should be enhanced since there is less time to redshift to today [15], which corresponds to enhanced indirect detection signals.

Catalyzed freeze-out.-In order for the catalyzed annihilation paradigm to work, there are four requirements: (1) The dark sector is nearly secluded. (2) The catalyst is long-lived $\left(\gtrsim 10^{-9} \mathrm{~s}\right)$. (3) The catalyst is slightly lighter than DM $\left(1<m_{\chi} / m_{A^{\prime}} \lesssim 2\right)$. (4) The annihilation channels shown in Fig. 1 are present.

The dark sector should be secluded so that the annihilation channels to SM particles freeze out before the catalyzed annihilation. The catalyst is lighter than DM and long-lived so that its number density is large and the $3 A^{\prime} \rightarrow 2 \chi$ process is not suppressed, which ensures that the catalyzed annihilation happens. If $A^{\prime}$ decays quickly, the paradigm recovers to the secluded DM regime [11,12]. In addition, the catalyzed annihilation will heat up the dark sector. For simplicity, we assume the dark sector could be scattered with SM particles intensely enough to maintain thermal equilibrium with SM particles.

We show in Fig. 2 a typical thermal history of dark matter that freezes out via catalyzed annihilation. For now, we are focused on the regime in which the mass ratio of dark matter and the catalyst $r \equiv m_{\chi} / m_{A^{\prime}}$ is no larger than

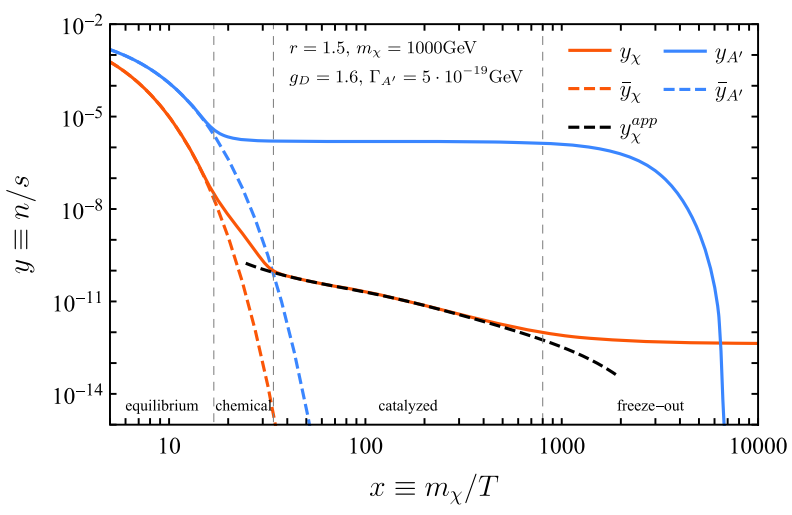

FIG. 2. Thermal evolution of DM $\chi$ (solid red) and the catalyst $A^{\prime}$ (solid blue). The dashed colored curves denote equilibrium yields $\bar{y} \equiv \bar{n} / s$, while the dashed black curve shows the approximate DM yield $y_{\chi}^{\text {app }} \equiv n_{\chi}^{\text {app }} / s$ during the catalyzed annihilation stage with $n_{\chi}^{\text {app }}=\sqrt{n_{A^{\prime}}^{3}\left\langle\sigma_{3} v^{2}\right\rangle /\left\langle\sigma_{2} v\right\rangle}$ from Eq. (4). The parameters are taken from the model presented below.

1.5. As shown in the figure, there are four stages in the thermal evolution.

Equilibrium stage: Both $\chi$ and $A^{\prime}$ stay in chemical equilibrium due to the number-changing processes in the dark sector. The dominant number-changing process is $3 A^{\prime} \rightarrow 2 \chi$ for $r \gtrsim 1.1$. Other processes with $\mathrm{DM}$ in the initial state, e.g., $\chi A^{\prime} A^{\prime} \rightarrow \chi A^{\prime}$, are suppressed and negligible, since $n_{\chi} \ll n_{A^{\prime}}$. In this stage, we have,

$$
n_{\chi} \simeq \bar{n}_{\chi}, \quad n_{A^{\prime}} \simeq \bar{n}_{A^{\prime}} .
$$

$n_{\chi, A^{\prime}}$ denote the number densities, and $\bar{n}_{\chi, A^{\prime}}$ are the equilibrium densities. In the nonrelativistic limit, we have $\bar{n}_{\chi, A^{\prime}}=g_{\chi, A^{\prime}}\left(m_{\chi, A^{\prime}} T / 2 \pi\right)^{3 / 2} e^{-m_{\chi, A^{\prime}} / T}$, where $g$ denotes number of internal degrees of freedom.

Chemical stage: $\chi$ and $A^{\prime}$ are chemically decoupled from equilibrium, but they can still maintain chemical equilibrium with each other via the $2 \chi \leftrightarrow 2 A^{\prime}$ process:

$$
n_{\chi} / \bar{n}_{\chi} \simeq n_{A^{\prime}} / \bar{n}_{A^{\prime}} .
$$

Catalyzed annihilation stage: As the rate of $2 A^{\prime} \rightarrow 2 \chi$ (inverse process of $2 \chi \rightarrow 2 A^{\prime}$ ) descends exponentially at low temperature, the $3 A^{\prime} \rightarrow 2 \chi$ process dominates it. The evolution of the DM number density is now controlled by the catalyzed annihilation, i.e., $2 \chi \rightarrow 2 A^{\prime}$ and $3 A^{\prime} \rightarrow 2 \chi$. Before freeze-out, the rates of the $2 \chi \rightarrow 2 A^{\prime}$ and $3 A^{\prime} \rightarrow 2 \chi$ reactions are much larger than the rate of change of $n_{\chi}$, as well as the Hubble rate and rates of other reactions [see Eq. (10)]. Thus, neglecting the subdominant terms, we get this approximate relation:

$$
\left\langle\sigma_{2} v\right\rangle n_{\chi}^{2} \simeq\left\langle\sigma_{3} v^{2}\right\rangle n_{A^{\prime}}^{3}
$$

We use $\left\langle\sigma_{2} v\right\rangle$ and $\left\langle\sigma_{3} v^{2}\right\rangle$ to denote the thermally averaged cross sections of $2 \chi \rightarrow 2 A^{\prime}$ and $3 A^{\prime} \rightarrow 2 \chi$, respectively. 
In this stage, since $y_{A^{\prime}} \equiv n_{A^{\prime}} / s$ is practically constant and $\left\langle\sigma_{2} v\right\rangle$ and $\left\langle\sigma_{3} v^{2}\right\rangle$ are polynomial functions of $T$, Eq. (4) indicates that $n_{\chi}$ is polynomially suppressed. The scaling of $n_{\chi}$ is similar to the scaling of the number density of the assisting particle after DM freeze-out in Ref. [21].

Freeze-out stage: As the Universe expands, the rate of the catalyzed annihilation decreases and dark matter freezes out.

The equilibrium stage ends when the rate of $3 A^{\prime} \rightarrow 2 \chi$ falls below the Hubble constant $H$. The temperature of departure from equilibrium $T_{c}$ can be determined approximately with

$$
\left\langle\sigma_{3} v^{2}\right\rangle \bar{n}_{A^{\prime}}^{3} \simeq H\left(\bar{n}_{A^{\prime}}+\bar{n}_{\chi}\right) .
$$

We note that the annihilation channels to SM particles or the $3 A^{\prime} \rightarrow 2 A^{\prime}$ process can also deplete dark sector particles and $T_{c}$ could be altered if these channels freeze out later. The ending of the chemical stage is insignificant since the freeze-out temperature $T_{f}$ and relic abundance can be estimated without it. Lastly, the catalyzed annihilation freezes out when the rate drops below $H . T_{f}$ is determined by

$$
\left\langle\sigma_{2} v\right\rangle n_{\chi}^{2} \simeq\left\langle\sigma_{3} v^{2}\right\rangle n_{A^{\prime}}^{3} \simeq H n_{\chi} .
$$

The relic abundance of DM can be estimated approximately in the same manner as WIMPs [15,33]:

$$
\Omega_{\chi}=\frac{m_{\chi} s_{0} H_{m}}{\rho_{c} s_{m}} \frac{\sqrt{g_{\star}, m}}{\sqrt{g_{\star, f}}} \frac{x_{f}}{\left\langle\sigma_{2} v\right\rangle},
$$

where $x_{f} \equiv m_{\chi} / T_{f}$. Since $x_{f}$ is dependent on $x_{c} \equiv m_{\chi} / T_{c}$, we solve for $x_{c}$ with Eq. (5) first. Simplifying Eq. (5), we find

$$
x_{c}=\frac{r}{2} \log \left[0.0024 \frac{g_{A^{\prime}}^{2} m_{\chi}^{4} M_{\mathrm{Pl}}\left\langle\sigma_{3} v^{2}\right\rangle_{c}}{g_{\star, c}^{1 / 2} r^{3} x_{c}}\right],
$$

where $M_{\mathrm{Pl}}$ is Planck mass. With $x_{c}$ determined in Eq. (8), we can solve for $n_{A^{\prime}}$. Substituting the result into Eq. (6), we get

$$
x_{f}=\left(1.2 \frac{g_{\star, f}^{2}}{g_{\star, c}^{9 / 4}} \frac{M_{\mathrm{Pl}}^{1 / 2}\left\langle\sigma_{2} v\right\rangle_{f}\left\langle\sigma_{3} v^{2}\right\rangle_{f}}{m_{\chi}\left\langle\sigma_{3} v^{2}\right\rangle_{c}^{3 / 2}} x_{c}^{6}\right)^{1 / 5} .
$$

The subscripts $m, c$, and $f$ in the equations above mark the temperatures $T=m_{\chi}, T_{c}$, and $T_{f}$, respectively, for the quantities, which include the entropy density $s$, the Hubble constant $H$, the effective degrees of freedom $g_{\star}$ [34], and the thermally averaged cross sections. Note that if $x_{c}$ is delayed due to annihilations to SM particles or $3 A^{\prime} \rightarrow 2 A^{\prime}$, Eqs. (9) and (8) should be modified to include these processes. Note also that DM will freeze out earlier in this case with a smaller relic abundance.

Based on the partial wave unitarity limit [35] $\sigma_{2} v \leq\left(4 \pi / m_{\chi}^{2} v\right)$, we can estimate the upper bound of DM mass from Eq. (7) for the catalyzed annihilation paradigm. With $x_{f} \gtrsim 100$, we deduce $m_{\chi} \lesssim 100 \mathrm{TeV}$. It is compelling to notice that, compared to SIMP dark matter that lives in the $\mathrm{MeV}$ scale [22], the $3 \rightarrow 2$ process can apply to such a heavy dark matter.

In order to study the thermal evolution and DM freezeout in a quantitative way, we turn to Boltzmann equations. As discussed above, we neglect the subdominant $3 \rightarrow 2$ annihilation channels, including $\chi A^{\prime} A^{\prime} \rightarrow \chi A^{\prime}, \chi \chi A^{\prime} \rightarrow A^{\prime} A^{\prime}$, $\chi \chi A^{\prime} \rightarrow \chi \chi$, and $\chi \chi \chi \rightarrow \chi A^{\prime}$ and assume $3 A^{\prime} \rightarrow 2 A^{\prime}$ is subdominant. If $A^{\prime}$ decays to SM particles, the Boltzmann equations reads

$$
\begin{aligned}
\dot{n}_{\chi}+3 H n_{\chi}= & -\left\langle\sigma_{2} v\right\rangle\left(n_{\chi}^{2}-\bar{n}_{\chi}^{2} \frac{n_{A^{\prime}}^{2}}{\bar{n}_{A^{\prime}}^{2}}\right) \\
& +\left\langle\sigma_{3} v^{2}\right\rangle\left(n_{A^{\prime}}^{3}-\bar{n}_{A^{\prime}}^{3} \frac{n_{\chi}^{2}}{\bar{n}_{\chi}^{2}}\right), \\
\dot{n}_{A^{\prime}}+3 H n_{A^{\prime}}= & +\left\langle\sigma_{2} v\right\rangle\left(n_{\chi}^{2}-\bar{n}_{\chi}^{2} \frac{n_{A^{\prime}}^{2}}{\bar{n}_{A^{\prime}}^{2}}\right) \\
& -\frac{3}{2}\left\langle\sigma_{3} v^{2}\right\rangle\left(n_{A^{\prime}}^{3}-\bar{n}_{A^{\prime}}^{3} \frac{n_{\chi}^{2}}{\bar{n}_{\chi}^{2}}\right) \\
& -\left\langle\Gamma_{A^{\prime}}\right\rangle\left(n_{A^{\prime}}-\bar{n}_{A^{\prime}}\right) .
\end{aligned}
$$

The yield $y_{\chi, A^{\prime}} \equiv n_{\chi, A^{\prime}} / s$ can be solved numerically and is shown in Fig. 2.

Mass ratio.-In the previous section, we concentrated on the mass ratio $r \leq 1.5$. In fact, the catalyzed annihilation paradigm can go beyond this limit. When the mass ratio is slightly larger than 1.5 , i.e., $3 m_{A^{\prime}}<2 m_{\chi},\left\langle\sigma_{3} v^{2}\right\rangle$ is exponentially suppressed as the temperature goes down:

$$
\left\langle\sigma_{3} v^{2}\right\rangle \propto e^{-(2 r-3) x / r},
$$

where $x \equiv m_{\chi} / T$. During the catalyzed annihilation period, with fewer DM particles produced via the $3 A^{\prime} \rightarrow 2 \chi$ process since the cross section is smaller, the DM number density shrinks more sharply. Consequently, the catalyzed annihilation freezes out much earlier.

As the mass ratio grows, when $r \lesssim 2$, it is intriguing to notice that the $4 A^{\prime} \rightarrow 2 \chi$ process may play a part in the catalyzed annihilation. To be specific, after a period of catalyzed annihilation governed by $2 \chi \rightarrow 2 A^{\prime}$ and $3 A^{\prime} \rightarrow 2 \chi$ as usual, there would be an extra stage of catalyzed annihilation predominated by $2 \chi \rightarrow 2 A^{\prime}$ and $4 A^{\prime} \rightarrow 2 \chi$ in which the nonsuppressed $4 A^{\prime} \rightarrow 2 \chi$ process takes over the role of converting $A^{\prime}$ to DM particles since the cross section of $3 A^{\prime} \rightarrow 2 \chi$ is exponentially suppressed [Eq. (11)], Similar to Eq. (4), we can deduce an approximate relation that holds in this stage: 


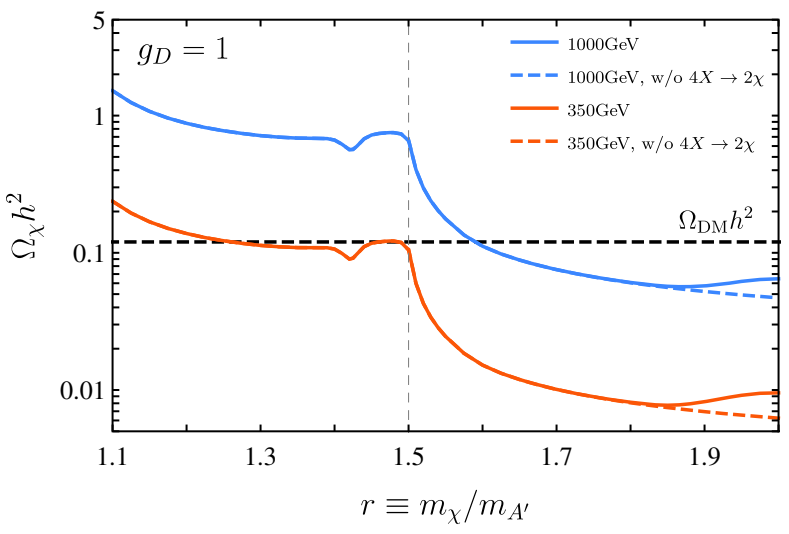

FIG. 3. Curves of relic abundance $\Omega_{\chi} h^{2}$ for $m_{\chi}=350 \mathrm{GeV}$ (solid red) and $m_{\chi}=1000 \mathrm{GeV}$ (solid blue) with respect to different mass ratios $r$. For dashed colored curves, the $4 A^{\prime} \rightarrow 2 \chi$ process is neglected. The dashed black curve denotes the observed DM relic abundance [36]. The parameters are taken from the model presented in below.

$$
\left\langle\sigma_{2} v\right\rangle n_{\chi}^{2} \simeq\left\langle\sigma_{4} v^{3}\right\rangle n_{A^{\prime}}^{4}
$$

where $\left\langle\sigma_{4} v^{3}\right\rangle$ denotes the thermally averaged cross section for $4 A^{\prime} \rightarrow 2 \chi$. The presence of $4 A^{\prime} \rightarrow 2 \chi$ is essential. If it is neglected, as discussed previously, $n_{\chi}$ shrinks sharply and dark matter freezes out early. Once $4 A^{\prime} \rightarrow 2 \chi$ takes charge, the sharp fall of $n_{\chi}$ is bent, and the polynomial suppression recovers [compared to Eq. (1)]:

$$
n_{\chi} \propto s^{2} \propto T^{6} \text {. }
$$

Thus, the catalyzed annihilation freezes out at later times, leading to enhanced DM relic abundance.

For an even larger mass ratio, we expect processes with more catalysts annihilating to two DM particles, e.g., $5 A^{\prime} \rightarrow 2 \chi$, to possibly play a role in the catalyzed annihilation, especially when the dark sector is strongly coupled.

We show in Fig. 3 the variation of DM relic abundance $\Omega_{\chi} h^{2}$ with different mass ratios. When the mass ratio passes the critical value of $1.5, \Omega_{\chi} h^{2}$ decreases rapidly. On the other hand, for $r \lesssim 2$, relic abundance is increased if the $4 A^{\prime} \rightarrow 2 \chi$ process is included.

A model.-The requirements for realization of the catalyzed annihilation presented in the "Catalyzed freeze-out" section can be easily met in many models. In this section, we simply present a dark photon model [37-41] with a Dirac fermion $\chi$ charged under a novel $U(1)^{\prime}$ gauge group with $A^{\prime}$ being the gauge field. The Lagrangian for the dark sector is

$\mathcal{L}_{\mathrm{DS}}=-\frac{1}{4} F_{\mu \nu}^{\prime} F^{\prime \mu \nu}+\frac{1}{2} m_{A^{\prime}}^{2} A_{\mu}^{\prime} A^{\prime \mu}+\bar{\chi}\left(i \not D-m_{\chi}\right) \chi$,

where $\not D=\not D-i g_{D} \not{ }^{\prime}$ and $g_{D}$ is the gauge coupling constant. The mass of the dark photon can be generated via the Higgs mechanism (or Stueckelberg mechanism

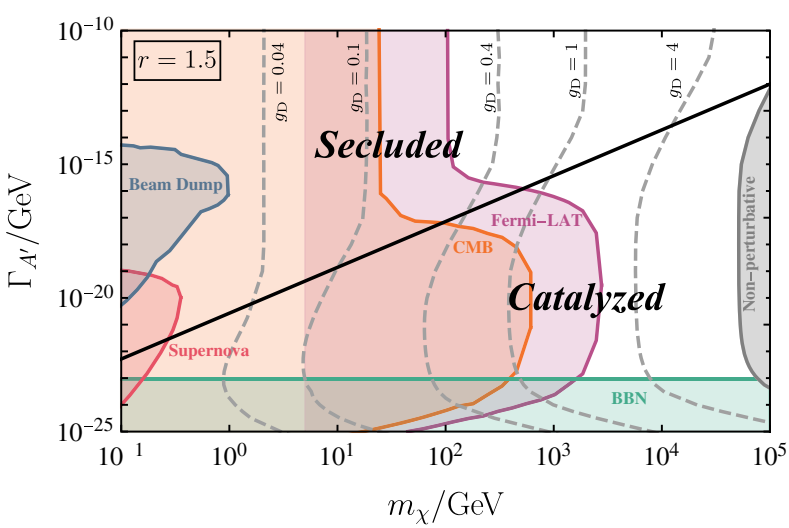

FIG. 4. Phase diagram and constraints for the dark photon model in the $\left(m_{\chi}, \Gamma_{A^{\prime}}\right)$ plane with $r=1.5$. The solid black curve marks the boundary of the secluded phase and the catalyzed phase of the model. Correct relic abundance can be reproduced for each point in the figure by varying the value of $g_{D}$. Especially, we show, in gray dashed curves, five different values of $g_{D}$ that reproduce a correct relic abundance. The nonperturbative region is shaded gray, while the color-shaded regions denote the bounds from various experiments and observations correspondingly.

$[42,43])$. We assume the dark Higgs boson is heavy and can be neglected. SM particles are neutral under the $U(1)^{\prime}$ gauge group. The dark photon can be kinetically mixed with an SM hypercharge field:

$$
\mathcal{L}_{\text {mix }}=-\frac{\epsilon}{2 \cos \theta_{W}} F_{\mu \nu}^{\prime} B^{\mu \nu},
$$

where $\epsilon$ is the mixing constant and $\theta_{W}$ denotes the Weinberg angle. $B^{\mu}$ is the SM hypercharge field. Therefore, the dark sector can communicate with SM particles via the mixing, and the dark photon $A^{\prime}$ can decay to SM particles. $\epsilon$ should be small so that the dark photon is long-lived and acts as the catalyst. Additionally, the kinetic mixing could not keep the dark sector in thermal equilibrium with SM since $\epsilon$ is small. In order to thermalize the dark sector, we need another portal for the dark sector to interact with SM particles, which might be the dark Higgs. Anyhow, we will not model this part and simply assume that the dark sector stays in thermal equilibrium before freeze-out.

We show in Fig. 4 different phases for the model in the calculation of the DM relic abundance. For short-lived dark photons, before dark matter freezes out, it simply stays in equilibrium with SM particles via the decay and inversedecay process. When DM particles annihilate into the dark photons, they immediately decay. This is the secluded phase of the model. On the other hand, when the dark photon width $\Gamma_{A^{\prime}}$ is small, the catalyzed annihilation emerges. It is a continuous shift, since the decay of the dark photon can occur during the catalyzed annihilation. When the dark photon decays after DM freeze-out, $\Omega_{\chi} h^{2}$ is independent with $\Gamma_{A^{\prime}}$. If $A^{\prime}$ is sufficiently long-lived 
$\left(\Gamma_{A^{\prime}} \lesssim 10^{-23} \mathrm{GeV}\right)$, it would come to dominate the energy density of the universe. When it decays, considerable entropy is produced and the DM abundance is diluted. This effect can help circumvent the upper bound of the DM mass [44-46] (see in Fig. 4). We show in Fig. 4 dashed gray curves for five different values of $g_{D}$ that reproduce the observed relic abundance.

The catalyzed annihilation paradigm is constrained by numerous terrestrial and celestial experiments and observations. The residual annihilation of $2 \chi \rightarrow 2 A^{\prime}$ after freezeout will distort the anisotropy of the cosmic microwave background since the decay products of $A^{\prime}$ are electrically charged particles [47-53]. Similarly, the signal of DM annihilation at present is detectable in indirect detection experiments [54-59]. The signal is enhanced compared to WIMPs with $\left\langle\sigma_{2} v\right\rangle_{0} \simeq 5 \times 10^{-25} \mathrm{~cm}^{3} \mathrm{~s}^{-1}$ for $s$-wave annihilation. We used bounds from the Fermi-LAT experiment to constrain our model in Fig. 4. The late time decay of $A^{\prime}$, on the other hand, is also stringently constrained by the cosmic microwave background [60-62], as well as big bang nucleosynthesis [63-65]. We note that these bounds can be evaded in models in which the catalyst decays into neutrinos or dark radiation [66-68]. Since the dark sector is highly secluded, the constraints on scattering with nucleons [69-72] are evaded.

For light dark photons, beam dump and fixed target experiments provide great sensitivity on the mixing coupling constant $\epsilon$ [73-76]. Also, many new experiments [77-80] have been proposed in recent years that focus on long-lived particles. In addition, the long-lived dark photon can enhance the cooling of supernova, and the constraints from SN 1987A [81-83] are widely discussed. These bounds on the dark photon model are considered and presented in Fig. 4.

Conclusion and discussion.-We proposed a novel paradigm for thermal relic dark matter yielding the observed relic abundance. The distinctive wisdom of the paradigm is that the dark matter freeze-out proceeds via catalyzed annihilation. We discussed in detail a scenario in which the catalyzed annihilation includes $2 \chi \rightarrow 2 A^{\prime}$ and $3 A^{\prime} \rightarrow 2 \chi$, where $\chi$ and $A^{\prime}$ are the dark matter and catalyst, respectively. The paradigm applies to a wide mass range of dark matter, from $1 \mathrm{MeV}$ to $100 \mathrm{TeV}$, with a unique thermal history compared to WIMPs and SIMPs. The paradigm also offers a rich phenomenology that includes the indirect DM search and long-lived particles.

We note that thermal decoupling effects can significantly modify dark matter relic abundance [16,30,84-87]. We leave this exploration to future work [88]. Additionally, catalyzed annihilation dominating DM abundance can go far beyond the reactions considered here and should be investigated further.

C. Y.X. would like to thank Yan-Fang Bai for encouragement. This work is supported by the National Science Foundation of China under Grants Nos. 11635001 and 11875072 .
*Corresponding author.

cyxing@pku.edu.cn

Corresponding author. shzhu@pku.edu.cn

[1] M. Bauer and T. Plehn, Yet Another Introduction to Dark Matter: The Particle Physics Approach, Lecture Notes in Physics Vol. 959 (Springer, New York, 2019).

[2] B. W. Lee and S. Weinberg, Cosmological Lower Bound on Heavy Neutrino Masses, Phys. Rev. Lett. 39, 165 (1977).

[3] G. Bertone, D. Hooper, and J. Silk, Particle dark matter: Evidence, candidates and constraints, Phys. Rep. 405, 279 (2005).

[4] G. Arcadi, M. Dutra, P. Ghosh, M. Lindner, Y. Mambrini, M. Pierre, S. Profumo, and F. S. Queiroz, The waning of the WIMP? A review of models, searches, and constraints, Eur. Phys. J. C 78, 203 (2018).

[5] L. Roszkowski, E. M. Sessolo, and S. Trojanowski, WIMP dark matter candidates and searches-current status and future prospects, Rep. Prog. Phys. 81, 066201 (2018).

[6] K. Griest and D. Seckel, Three exceptions in the calculation of relic abundances, Phys. Rev. D 43, 3191 (1991).

[7] J. R. Ellis, T. Falk, and K. A. Olive, Neutralino-Stau coannihilation and the cosmological upper limit on the mass of the lightest supersymmetric particle, Phys. Lett. B 444, 367 (1998).

[8] R. T. D’Agnolo, C. Mondino, J. T. Ruderman, and P.-J. Wang, Exponentially light dark matter from coannihilation, J. High Energy Phys. 08 (2018) 079.

[9] F. D'Eramo and J. Thaler, Semi-annihilation of dark matter, J. High Energy Phys. 06 (2010) 109.

[10] R. T. D'Agnolo and J. T. Ruderman, Light Dark Matter from Forbidden Channels, Phys. Rev. Lett. 115, 061301 (2015).

[11] M. Pospelov, A. Ritz, and M. B. Voloshin, Secluded WIMP dark matter, Phys. Lett. B 662, 53 (2008).

[12] N. Arkani-Hamed, D. P. Finkbeiner, T. R. Slatyer, and N. Weiner, A theory of dark matter, Phys. Rev. D 79, 015014 (2009).

[13] J. L. Feng and J. Kumar, The WIMPless Miracle: DarkMatter Particles without Weak-Scale Masses or Weak Interactions, Phys. Rev. Lett. 101, 231301 (2008).

[14] G. Belanger and J.-C. Park, Assisted freeze-out, J. Cosmol. Astropart. Phys. 03 (2012) 038.

[15] J. A. Dror, E. Kuflik, and W. H. Ng, Codecaying Dark Matter, Phys. Rev. Lett. 117, 211801 (2016).

[16] R. T. D’Agnolo, D. Pappadopulo, and J. T. Ruderman, Fourth Exception in the Calculation of Relic Abundances, Phys. Rev. Lett. 119, 061102 (2017).

[17] M. Garny, J. Heisig, M. Hufnagel, B. Lülf, and S. Vogl, Conversion-driven freeze-out: Dark matter genesis beyond the WIMP paradigm, Proc. Sci., CORFU2018 (2019) 092 [arXiv:1904.00238].

[18] T. N. Maity and T. S. Ray, Exchange driven freeze out of dark matter, Phys. Rev. D 101, 103013 (2020).

[19] E. D. Kramer, E. Kuflik, N. Levi, N. J. Outmezguine, and J. T. Ruderman, Heavy Thermal Relics from Zombie Collisions, Phys. Rev. Lett. 126, 081802 (2021).

[20] U. K. Dey, T. N. Maity, and T. S. Ray, Light dark matter through assisted annihilation, J. Cosmol. Astropart. Phys. 03 (2017) 045. 
[21] J. M. Cline, H. Liu, T. Slatyer, and W. Xue, Enabling forbidden dark matter, Phys. Rev. D 96, 083521 (2017).

[22] Y. Hochberg, E. Kuflik, T. Volansky, and J. G. Wacker, Mechanism for Thermal Relic Dark Matter of Strongly Interacting Massive Particles, Phys. Rev. Lett. 113, 171301 (2014).

[23] Y. Hochberg, E. Kuflik, H. Murayama, T. Volansky, and J. G. Wacker, Model for Thermal Relic Dark Matter of Strongly Interacting Massive Particles, Phys. Rev. Lett. 115, 021301 (2015).

[24] N. Bernal and X. Chu, $\mathbb{Z}_{2}$ SIMP dark matter, J. Cosmol. Astropart. Phys. 01 (2016) 006.

[25] N. Bernal, X. Chu, and J. Pradler, Simply split strongly interacting massive particles, Phys. Rev. D 95, 115023 (2017).

[26] J. Smirnov and J. F. Beacom, New Freezeout Mechanism for Strongly Interacting Dark Matter, Phys. Rev. Lett. 125, 131301 (2020).

[27] T. Engel and P. Reid, Physical Chemistry, 2nd ed. (Pearson Education, Upper Saddle River, NJ; Harlow, 2009).

[28] G. Rothenberg, Catalysis, 2nd ed. (Wiley, Newark, 2017).

[29] R. H Petrucci, General Chemistry: Principles and Modern Applications, 8th ed. (Prentice Hall, Upper Saddle River, NJ, 2002).

[30] P. J. Fitzpatrick, H. Liu, T. R. Slatyer, and Y.-D. Tsai, New pathways to the relic abundance of vector-portal dark matter, arXiv:2011.01240.

[31] A. D. Dolgov, New old mechanism of dark matter burning, arXiv: 1705.03689.

[32] M. Pospelov, Particle physics catalysis of thermal Big Bang Nucleosynthesis, Phys. Rev. Lett. 98, 231301 (2007).

[33] E. W. Kolb and M. S. Turner, The early universe, Front. Phys. 69, 1 (1990), https://inspirehep.net/literature/299778.

[34] We ignore the differences between effective entropy degrees of freedom $g_{\star, s}$ and effective energy degrees of freedom $g_{\star}$ as in Ref. [15].

[35] K. Griest and M. Kamionkowski, Unitarity Limits on the Mass and Radius of Dark Matter Particles, Phys. Rev. Lett. 64, 615 (1990).

[36] N. Aghanim et al. (Planck Collaboration), Planck 2018 results. VI. Cosmological parameters, Astron. Astrophys. 641, A6 (2020).

[37] B. Holdom, Two U(1)'s and epsilon charge shifts, Phys. Lett. 166B, 196 (1986).

[38] M. Raggi and V. Kozhuharov, Results and perspectives in dark photon physics, Riv. Nuovo Cimento 38, 449 (2015).

[39] M. Bauer, P. Foldenauer, and J. Jaeckel, Hunting all the hidden photons, J. High Energy Phys. 07 (2018) 094.

[40] J. Lao, C. Cai, Z.-H. Yu, Y.-P. Zeng, and H.-H. Zhang, Fermionic and scalar dark matter with hidden U(1) gauge interaction and kinetic mixing, Phys. Rev. D 101, 095031 (2020).

[41] M. Fabbrichesi, E. Gabrielli, and G. Lanfranchi, The dark photon, arXiv:2005.01515.

[42] E. C. G. Stueckelberg, Interaction energy in electrodynamics and in the field theory of nuclear forces, Helv. Phys. Acta 11, 225 (1938).

[43] H. Ruegg and M. R.-Altaba, The Stueckelberg field, Int. J. Mod. Phys. A 19, 3265 (2004).
[44] G. Gelmini, P. Gondolo, A. Soldatenko, and C. E. Yaguna, The effect of a late decaying scalar on the neutralino relic density, Phys. Rev. D 74, 083514 (2006).

[45] P. S. Bhupal Dev, R. N. Mohapatra, and Y. Zhang, Naturally stable right-handed neutrino dark matter, J. High Energy Phys. 11 (2016) 077.

[46] A. Berlin, D. Hooper, and G. Krnjaic, PeV-scale dark matter as a thermal relic of a decoupled sector, Phys. Lett. B 760, 106 (2016).

[47] N. Padmanabhan and D. P. Finkbeiner, Detecting dark matter annihilation with $\mathrm{CMB}$ polarization: Signatures and experimental prospects, Phys. Rev. D 72, 023508 (2005).

[48] S. Galli, F. Iocco, G. Bertone, and A. Melchiorri, CMB constraints on dark matter models with large annihilation cross-section, Phys. Rev. D 80, 023505 (2009).

[49] M. Kawasaki, K. Nakayama, and T. Sekiguchi, CMB constraint on dark matter annihilation after Planck 2015, Phys. Lett. B 756, 212 (2016).

[50] T. R. Slatyer, Indirect dark matter signatures in the cosmic dark ages. I. Generalizing the bound on s-wave dark matter annihilation from Planck results, Phys. Rev. D 93, 023527 (2016).

[51] H. Liu, T. R. Slatyer, and J. Zavala, Contributions to cosmic reionization from dark matter annihilation and decay, Phys. Rev. D 94, 063507 (2016).

[52] N. Aghanim et al. (Planck Collaboration), Planck 2018 results. V. CMB power spectra and likelihoods, Astron. Astrophys. 641, A5 (2020).

[53] J. Cang, Y. Gao, and Y.-Z. Ma, Probing dark matter with future CMB measurements, Phys. Rev. D 102, 103005 (2020).

[54] O. Adriani et al. (PAMELA Collaboration), Cosmic-Ray Positron Energy Spectrum Measured by PAMELA, Phys. Rev. Lett. 111, 081102 (2013).

[55] M. Ackermann et al. (Fermi-LAT Collaboration), Searching for Dark Matter Annihilation from Milky Way Dwarf Spheroidal Galaxies with Six Years of Fermi Large Area Telescope Data, Phys. Rev. Lett. 115, 231301 (2015).

[56] A. Albert et al. (Fermi-LAT, DES Collaborations), Searching for dark matter annihilation in recently discovered Milky Way satellites with Fermi-LAT, Astrophys. J. 834, 110 (2017).

[57] M. Aguilar et al. (AMS Collaboration), Antiproton Flux, Antiproton-to-Proton Flux Ratio, and Properties of Elementary Particle Fluxes in Primary Cosmic Rays Measured with the Alpha Magnetic Spectrometer on the International Space Station, Phys. Rev. Lett. 117, 091103 (2016).

[58] S. Profumo, F. S. Queiroz, J. Silk, and C. Siqueira, Searching for Secluded Dark Matter with H.E.S.S., Fermi-LAT, and Planck, J. Cosmol. Astropart. Phys. 03 (2018) 010.

[59] H. Abdallah et al. (HESS Collaboration), Search for $\gamma$-Ray Line Signals from Dark Matter Annihilations in the Inner Galactic Halo from 10 Years of Observations with H.E.S.S., Phys. Rev. Lett. 120, 201101 (2018).

[60] X.-L. Chen and M. Kamionkowski, Particle decays during the cosmic dark ages, Phys. Rev. D 70, 043502 (2004).

[61] S. K. Acharya and R. Khatri, New CMB spectral distortion constraints on decaying dark matter with full evolution of 
electromagnetic cascades before recombination, Phys. Rev. D 99, 123510 (2019).

[62] S. K. Acharya and R. Khatri, CMB anisotropy and BBN constraints on pre-recombination decay of dark matter to visible particles, J. Cosmol. Astropart. Phys. 12 (2019) 046.

[63] M. Kawasaki, K. Kohri, and T. Moroi, Big-Bang nucleosynthesis and hadronic decay of long-lived massive particles, Phys. Rev. D 71, 083502 (2005).

[64] K. Jedamzik, Big bang nucleosynthesis constraints on hadronically and electromagnetically decaying relic neutral particles, Phys. Rev. D 74, 103509 (2006).

[65] M. Kawasaki, K. Kohri, T. Moroi, and Y. Takaesu, Revisiting big-bang nucleosynthesis constraints on long-lived decaying particles, Phys. Rev. D 97, 023502 (2018).

[66] K. Ichiki, M. Oguri, and K. Takahashi, WMAP Constraints on Decaying Cold Dark Matter, Phys. Rev. Lett. 93, 071302 (2004).

[67] V. Poulin, P. D. Serpico, and J. Lesgourgues, A fresh look at linear cosmological constraints on a decaying dark matter component, J. Cosmol. Astropart. Phys. 08 (2016) 036.

[68] A. Nygaard, T. Tram, and S. Hannestad, Updated constraints on decaying cold dark matter, J. Cosmol. Astropart. Phys. 05 (2021) 017.

[69] X. Cui et al. (PandaX-II Collaboration), Dark Matter Results From 54-Ton-Day Exposure of PandaX-II Experiment, Phys. Rev. Lett. 119, 181302 (2017).

[70] E. Aprile et al. (XENON Collaboration), Dark Matter Search Results from a One Ton-Year Exposure of XENON1T, Phys. Rev. Lett. 121, 111302 (2018).

[71] R. K. Leane and J. Smirnov, Exoplanets as Sub-GeV Dark Matter Detectors, Phys. Rev. Lett. 126, 161101 (2021).

[72] R. K. Leane, T. Linden, P. Mukhopadhyay, and N. Toro, Celestial-body focused dark matter annihilation throughout the Galaxy, Phys. Rev. D 103, 075030 (2021).

[73] F. Bergsma et al. (CHARM Collaboration), A search for decays of heavy neutrinos in the mass range $0.5-\mathrm{GeV}$ to 2.8 GeV, Phys. Lett. 166B, 473 (1986).

[74] J. D. Bjorken, S. Ecklund, W. R. Nelson, A. Abashian, C. Church, B. Lu, L. W. Mo, T. A. Nunamaker, and P. Rassmann, Search for neutral metastable penetrating particles produced in the SLAC beam dump, Phys. Rev. D 38, 3375 (1988).
[75] M. Davier and H. Nguyen Ngoc, An unambiguous search for a light Higgs boson, Phys. Lett. B 229, 150 (1989).

[76] J. Blümlein and J. Brunner, New exclusion limits on dark gauge forces from proton Bremsstrahlung in beam-dump data, Phys. Lett. B 731, 320 (2014).

[77] M. Anelli et al. (SHiP Collaboration), A facility to search for hidden particles (SHiP) at the CERN SPS, arXiv: 1504.04956.

[78] J. L. Feng, I. Galon, F. Kling, and S. Trojanowski, Forward search experiment at the LHC, Phys. Rev. D 97, 035001 (2018).

[79] A. Berlin, S. Gori, P. Schuster, and N. Toro, Dark sectors at the Fermilab seaquest experiment, Phys. Rev. D 98, 035011 (2018).

[80] P. H. Adrian et al. (HPS Collaboration), Search for a dark photon in electroproduced $e^{+} e^{-}$pairs with the Heavy Photon Search experiment at JLab, Phys. Rev. D 98, 091101 (2018).

[81] J. H. Chang, R. Essig, and S. D. McDermott, Revisiting supernova 1987A constraints on dark photons, J. High Energy Phys. 01 (2017) 107.

[82] J. H. Chang, R. Essig, and Samuel D. McDermott, Supernova 1987A constraints on sub-GeV dark sectors, millicharged particles, the QCD axion, and an axion-like particle, J. High Energy Phys. 09 (2018) 051.

[83] A. Sung, H. Tu, and M.-R. Wu, New constraint from supernova explosions on light particles beyond the Standard Model, Phys. Rev. D 99, 121305(R) (2019).

[84] E. Kuflik, M. Perelstein, N. Rey-Le Lorier, and Y.-D. Tsai, Elastically Decoupling Dark Matter, Phys. Rev. Lett. 116, 221302 (2016).

[85] D. Pappadopulo, J. T. Ruderman, and G. Trevisan, Dark matter freeze-out in a nonrelativistic sector, Phys. Rev. D 94, 035005 (2016).

[86] M. Farina, D. Pappadopulo, J. T. Ruderman, and G. Trevisan, Phases of cannibal dark matter, J. High Energy Phys. 12 (2016) 039.

[87] E. Kuflik, M. Perelstein, N. Rey-Le Lorier, and Y.-D. Tsai, Phenomenology of ELDER dark matter, J. High Energy Phys. 08 (2017) 078.

[88] C.-Y. Xing and S.-h. Zhu (to be published). 